Agronomía Costarricense 42(2): 43-57. ISSN:0377-9424 / 2018

www.mag.go.cr/rev_agr/index.html www.cia.ucr.ac.cr

\title{
EVALUACIÓN DE Si Y Ca FOLIAR EN EL CRECIMIENTO Y TOLERANCIA DE COMPLEJO DE NECROSIS FOLIAR EN PALMA ACEITERA
}

\author{
Gabriel Garbanzo ${ }^{1 / *}$, Eloy Molina*, Gilberto Cabalceta*, Floria Ramírez** \\ Palabras clave: Silicio; calcio; palma aceitera; concentración foliar; nutrición; enfermedades; vivero. \\ Keywords: Silicon; calcium; oil palm; foliar concentration; plant nutrition; diseases; nursery.
}

Recibido: 02/03/17

Aceptado: $13 / 07 / 17$

\section{RESUMEN}

Se realizaron aplicaciones de distintas fuentes de $\mathrm{Si}$ y $\mathrm{Ca}$ foliar en vivero de palma aceitera y se evaluó el efecto de la severidad del Complejo de Necrosis Foliar (CNF) en el Pacífico Sur de Costa Rica. Se utilizaron 4 fertilizantes foliares de $\mathrm{Ca}, 3$ fuentes de $\mathrm{Si}$ y una fuente de $\mathrm{Ca}$ con fitoalexinas. El diseño experimental fue de bloques completos al azar con 9 tratamientos y 4 repeticiones. Se evaluó a los 85, 130, 175, 220, 265 y 300 días después de siembra (dds) el crecimiento morfológico de las plantas y el porcentaje de severidad de CNF en las hojas. A los 175, 220, 265 y 300 dds se evaluó la concentración foliar de N, P, K, Ca, Mg, S, $\mathrm{Fe}, \mathrm{Zn}, \mathrm{Mn}, \mathrm{Cu}, \mathrm{B}$ y Si. Las aplicaciones de Si y $\mathrm{Ca}$ foliar no mostraron un efecto significativo en la tolerancia al CNF en el tiempo. No se encontraron diferencias significativas en las concentraciones foliares de $\mathrm{Si}$ y $\mathrm{Ca}$. El tratamiento SupCu proporcionó mayor concentración de $\mathrm{Cu}$ en las plantas de palma aceitera y redujo significativamente la severidad de CNF. Además, se encontró que productos con contenidos de $\mathrm{K}$ y $\mathrm{P}$ en forma de fosfitos pueden aumentar el vigor en las plantas. Se concluyó que los fertilizantes foliares con $\mathrm{Ca}$ y $\mathrm{Si}$ no fueron eficaces para el

\footnotetext{
1 Autor para correspondencia. Correo electrónico: juan.garbanzo@ucr.ac.cr Universidad de Costa Rica, Centro de Investigaciones Agronómicas, Costa Rica.
}

\begin{abstract}
$\mathrm{Si}$ and Ca foliar evaluation in the growth and tolerance to foliar necrosis complex in oil palm nurseries. The evaluation of different sources of silicon and Calcium foliar in the growth and tolerance of diseases in oil palm nurseries it was evaluate in the pacific south of Costa Rica. Different sources of foliar Si and Ca were applied to oil palm nurseries and it was evaluated the effect in foliar necrosis complex (Complejo de Necrosis Foliar in Spanish, CNF) severity. The experiment consisted to aspersion on leaves of four Ca sources, three $\mathrm{Si}$ sources and one $\mathrm{Ca}$ source with phytoalexins. The experiment design was a randomized complete block with 9 treatments and 4 repetitions. The experiment variables evaluated were the morphological growth and \% of CNF at 85, 130, 175, 220, 265 and 300 days after planting (dds). Also, it was evaluated the foliar concentration of $\mathrm{N}, \mathrm{P}, \mathrm{K}, \mathrm{Ca}, \mathrm{Mg}, \mathrm{S}, \mathrm{Fe}$, $\mathrm{Zn}, \mathrm{Mn}, \mathrm{Cu}, \mathrm{B}$ and $\mathrm{Si}$ at $175,220,265$ y 300 dds. It was found that the application of foliar $\mathrm{Si}$ and $\mathrm{Ca}$ did not show a significant effect on $\mathrm{CNF}$ tolerance over time. The foliar Si concentration and $\mathrm{Ca}$ did not show significant difference. The $\mathrm{SupCu}$ treatment increased the concentration of $\mathrm{Cu}$ in the plant and this decreased significantly
\end{abstract}

\footnotetext{
** Palma Tica S.A. Departamento de Investigación y Desarrollo, Puntarenas, Costa Rica.
} 
control del CNF y que productos con $\mathrm{Cu}$ pueden aumentar la tolerancia a este complejo.

\section{INTRODUCCIÓN}

El Si es un nutrimento no esencial para las plantas, se caracteriza por ser el segundo elemento de mayor abundancia y disperso en la corteza terrestre, superado por el oxgeno (Quero 2008, Seron 2004). El Si representa entre 0,1\% y $10 \%$ del peso seco de las plantas superiores, por lo que se encuentra en mayor porción en monocotiledóneas que en dicotiledóneas (Epstein 1999, Aguirre et al. 2007). Este nutrimento puede llegar a concentrarse en las plantas en cantidades superiores a $\mathrm{Ca}, \mathrm{Mg}, \mathrm{P}, \mathrm{y} \mathrm{K}$, principalmente en Poaceae (Epstein 1999 y Ahmed et al. 2012). El Si se absorbe de manera energéticamente pasiva de la solución del suelo en forma de ácido monosilícico y es depositado en la pared celular en forma de polímero hidratado o sílice amorfo (Ma y Yamaji 2006, Carneiro et al. 2010 citado por Lobo 2013), que se impregnan en una fina capa de 2,5 um (Epstein 1999, Pilon et al. 2013). Esta forma es ligeramente soluble en las hojas y corteza, lo cual convierte el ácido silícico en sílice coloidal y, posteriormente, en gel de sílice ("Opalo o fitólitos" $\mathrm{SiO}_{2}-\mathrm{nH}_{2} \mathrm{O}$ ), mediante un proceso de polimeración o policondensación (Savant et al. 1997 y Da Costa et al. 2005, Barros et al. 2010).

El Ca es un catión divalente esencial para el desarrollo de las plantas superiores que se caracteriza por estar presente en rocas sedimentarias que emergieron de los levantamientos de the CNF. Also, it was found that sources with $\mathrm{K}$ and $\mathrm{P}$ in form of phosphites increased probably the growth of the plant of oil palm. It was concluded that the foliar fertilizers with $\mathrm{Ca}$ and $\mathrm{Si}$ were not effective for CNF control, but the $\mathrm{Cu}$ sources, these probably could increase the tolerance to $\mathrm{CNF}$.

los pisos marinos de la corteza terrestre (Hardy 1970, Buol 2003, Jaramillo 2011). Se encuentra entre un 0,1 y $5 \%$ del peso seco de las plantas superiores, con una movilidad limitada en las células (Marschner y Marschner 2012). Este nutrimento se absorbe en forma pasiva, por lo que es transportado por la corriente transpiratoria de la planta hacia las células del tallo y hojas (Nath 2015). Posee un papel importante en la estructura de la planta, debido a que da estabilidad en la pared celular, promueve formación de pectatos y oxalatos, participa en la división celular, en regulación estomática, en procesos enzimáticos y hormonales, entre otros (Benton 1997, Mengel et al. 2001, Taiz y Zeiger 2006).

El Si y Ca se encuentran involucrados en la tolerancia al estrés producido por problemas bióticos. Estudios realizados con Si evidenciaron su importancia en la activación de genes que proveen la producción de enzimas, que son correlacionadas con los mecanismos de defensa de las plantas (Chérif et al. 1994, Bélanger et al. 1995, Fawe 1998 citado por Tsai et al. 2004, Liang et al. 2015). Por otro lado, tanto en Si y $\mathrm{Ca}$ se acumulan en la pared celular, aspecto que previene el ingreso de haustorios en las células (Datnoff et al. 2007, Ma y Yamaji 2006). Al estar fortalecida la pared celular, la segregación de enzimas liberadas por los hongos tampoco dañan la pared (Fauteux et al. 2005), lo cual evita el ingreso del patógeno y dificulta el 
proceso de infección en las plantas (Epstein 1999, Arauz 2011, Anderson 2012).

La fertilización foliar es una herramienta utilizada en la actualidad para suplir nutrimentos complementarios a los requerimientos en la nutrición de las plantas. La absorción foliar de nutrimentos se realiza por diversos mecanismos en los tejidos, lo cual inicia la penetración a través de la capa cerosa de la cutícula en forma de difusión pasiva (Marschner y Marschner 2012, Nath 2015). Luego, estos son guiados hacia unas prolongaciones llamadas ectositodos, que conectan la parte externa de la cutícula hasta las paredes primarias celulares (matriz). Posteriormente, los nutrimentos son depositados sobre la pared celular y movidos mediante una ruta apoplástica hacia otras paredes celulares vecinas (Mengel et al. 2001, Taiz y Zeiger 2006). La velocidad de absorción de los diferentes nutrimentos varía según el intercambio catiónico y la valencia del ión aplicados en la hoja, los iones monovalentes penetran con mayor facilidad que los iones de mayores valencias (Trinidad y Aguilar 1999).

Los viveros de palma aceitera generalmente son afectados por enfermedades foliares, que reducen el crecimiento y el vigor de las plantas entre un 10 - 20\% (Ulloa y Ramírez 2011, Ulloa y Serrano 2012). Investigaciones realizadas por Turner y Bull (1967), Renard y Quillec (1979), Turner (1981) citados en Ulloa y Serrano (2012), reportaron la presencia de agentes causales que provocan lesiones importantes en las láminas foliares en vivero, entre estos se encuentran: Botryodiplodia sp., Melanconium sp., Colletotrichum sp. [Glomerella sp.] y Curvularia sp. Investigaciones en Costa Rica encontraron Colletotrichum gloeosporioides, que afecta láminas foliares (Ortiz y Fernández 2000). Sin embargo, las agentes causales de enfermedades reportadas en vivero pueden ser ocasionales según el crecimiento de la planta, condiciones climatológicas y manejo.
El objetivo de esta investigación fue evaluar el efecto de distintas fuentes de $\mathrm{Si}$ y $\mathrm{Ca}$ rociados en hojas para evaluar el crecimiento y severidad de Complejo Necrosis Foliar en viveros de palma aceitera.

\section{MATERIALES Y MÉTODOS}

La investigación se llevó cabo en los viveros de la Compañía Palma Tica S.A., ubicada en Coto 47 en el cantón de Corredores en Puntarenas, Costa Rica. Esta zona se encuentra en la llanura aluvial de Coto-Corredores, con una elevación aproximada entre $24-30 \mathrm{msnm}$. La precipitación promedio anual registrada varía entre $3800-4500 \mathrm{~mm}$. Se procedió a medir las condiciones climatológicas durante el periodo de evaluación, que comprendió de julio de 2014 a marzo 2015, se registró una precipitación total $2051 \mathrm{~mm}$. La temperatura promedio durante el periodo fue Max: $36,4^{\circ} \mathrm{C}$ y Min: $26,5^{\circ} \mathrm{C}$, con una humedad relativa promedio de $87,4 \%$ y una radiación solar promedio de 377,2 Wat.m ${ }^{-2}$.

Se ubicó una zona de vivero donde fue frecuente la presencia de enfermedades foliares, homogénea entre sus dimensiones con un área de $4752 \mathrm{~m}^{2}$. Se utilizaron 3480 bolsas con suelo aluvial Inceptisol, las cuales se utilizan comúnmente en vivero, cada una contenía $20 \mathrm{~L}$ de suelo. Las bolsas fueron distribuidas en campo, de manera que en los boquetes presentaran filas de 30 plantas, posteriormente, se etiquetaron con números continuos $(1-1080)$ y se dejaron 2 filas (plantas bordes) entre las etiquetadas. Las bolsas estuvieron 60 días en reposo mientras crecían las plantas de etapa de pre-vivero.

Se utilizaron fertilizantes foliares con concentraciones superiores a $9 \%$ de $\mathrm{Si} \mathrm{y} 7 \%$ de $\mathrm{Ca}$ que fueran soluciones concentradas para mejorar su solubilidad y evitar obstaculizar las boquillas de aplicación. Se seleccionaron 4 fuentes foliares de $\mathrm{Ca}, 3$ fuentes de $\mathrm{Si}$ y una fuente de $\mathrm{Ca}$ con fitoalexinas (Cuadro 1). 
Cuadro 1. Características químicas de fertilizantes foliares con Ca y Si asperjados en vivero de E. guineensis en la región Coto, Costa Rica.

\begin{tabular}{l|ccccccccc}
\hline \multirow{2}{*}{ Productos } & \multicolumn{7}{c}{$\% \mathrm{p} / \mathrm{v}$} & & \\
\cline { 2 - 9 } & $\mathrm{CaO}$ & $\mathrm{N}$ & $\mathrm{SiO}_{2}$ & $\mathrm{~K}_{2} \mathrm{O}$ & $\mathrm{B}$ & $\mathrm{MgO}$ & $\mathrm{S}$ & $\mathrm{Cu}$ & $\mathrm{P}_{2} \mathrm{O}_{5}$ \\
\hline Cal56 & 56,0 & 6,9 & - & - & - & - & - & - & - \\
Supa Cobre & 30,6 & - & - & - & - & - & - & 5,6 & - \\
Polisulfuro de calcio & 7,5 & - & - & - & - & - & 15,0 & - & - \\
Calcimax & 14,3 & - & - & - & 0,64 & - & - & - & - \\
Sifol Plus & 2,5 & - & 20,0 & 9,0 & - & - & - & 1,3 & 18,0 \\
Firmeza KCS & 9,8 & 1,8 & 11,0 & 6,0 & - & - & 4,1 & - & - \\
Stan SKH & - & - & 20,0 & 18,0 & - & - & - & - & - \\
Alexin & 4,3 & - & - & 10,1 & 0,25 & 1,68 & - & - & - \\
\hline
\end{tabular}

Antes de la siembra se aplicó una dosis de herbicida para eliminar la maleza presente en el área de vivero y las bolsas, con el fin de aligerar la siembra de las plantas. Asimismo, se realizó un análisis químico para determinar el estado nutricional del suelo en las bolsas
(Cuadro 2) y 2 semanas después se sembraron plántulas de E. guineensis de la variedad Compacta x Gana, que fueron sembradas en Jiffi en la etapa de pre-vivero, donde pasaron 2 meses de establecimiento bajo condiciones controladas.

Cuadro 2. Contenido de nutrimentos de suelo en bolsa utilizado para prueba foliar en etapa de vivero de palma aceitera.

\begin{tabular}{|c|c|c|c|c|c|c|c|c|c|c|c|c|c|}
\hline \multirow{2}{*}{$\begin{array}{c}\mathrm{ID}^{+} \\
\text {Mehlich } 3\end{array}$} & \multirow{2}{*}{$\begin{array}{c}\mathrm{pH} \\
\mathrm{H}_{2} \mathrm{O}\end{array}$} & \multicolumn{5}{|c|}{$\operatorname{cmol}(+) \cdot 1^{-1}$} & \multirow{2}{*}{$\begin{array}{l}\% \\
\text { SA }\end{array}$} & \multicolumn{6}{|c|}{$\mathrm{m} . \mathrm{l}^{-1}$} \\
\hline & & Acidez $(\mathrm{KCl})$ & $\mathrm{Ca}$ & $\mathrm{Mg}$ & K & CICE & & $\mathrm{P}$ & $\mathrm{Zn}$ & $\mathrm{Cu}$ & $\mathrm{Fe}$ & $\mathrm{Mn}$ & $\mathrm{Si}^{*}$ \\
\hline $\mathrm{NC}$ & 5,5 & 0,5 & 4,0 & 1,1 & 0,2 & 5,0 & & 15 & 1,7 & 0,4 & 10 & 4 & 200 \\
\hline Suelo inicio & 6,38 & 0,34 & 36,0 & 4,11 & 0,49 & 40,9 & 0,83 & 18,7 & 2,44 & 16,3 & 314 & 35 & 176 \\
\hline
\end{tabular}

+ Solución extractora Mehlich 3, laboratorio suelos y foliares Palma Tica S.A.; NC = nivel crítico para Inceptisoles según Cabalceta y Molina (2006). CICE $=$ Capacidad de intercambio de Cationes Efectiva $=$ Acidez $+\mathrm{Ca}+\mathrm{Mg}+\mathrm{K}$. * Metodología de extracción con ácido acético según Lobo (2013).

Se realizó el mismo manejo agronómico utilizado para viveros, con excepción de la nutrición y el control de enfermedades foliares. La fórmula que se utilizó en vivero corresponde a $15,50-9,76-15,50-4,00-3,20(\mathrm{~S})-0,30$ (B), constituidas por las fuentes de nitrato de amonio (NAM), Microessentials (SZ), cloruro de potasio $(\mathrm{KCl})$, kiesierita y granubor. Esta fórmula se calculó según los requisitos determinados mediante la curva de absorción propuesta por Ramírez y Muñoz (2010). La fertilización se aplicó cada 15 días de forma granular en el área superficial de la bolsa, distanciados del bulbo para no producir pérdida de agua en la planta por difusión.

Posteriormente, se procedió a calcular una dosis estándar dentro del rango de las recomendaciones pre-establecidas para los productos y que fue ajustada al plan de aspersión 
definido en el vivero de la Compañía Palma Tica S.A. en conjunto con el crecimiento de las plantas. Las aspersiones se realizaron de la siguiente manera: entre los 85 y 135 días después de sembrado (dds) aplicaron $0,61 . \mathrm{ha}^{-1} \mathrm{de}$ cada producto, de 150 y 200 dds $1,051 . \mathrm{ha}^{-1}$ y a partir de los 215 dds hasta terminar la etapa de vivero 1,5 1.ha-1 (Cuadro 3). Estas aplicaciones se realizaron continuamente cada 15 días desde la primera semana de siembra en vivero.

Cuadro 3. Dosis utilizadas de fertilizantes foliares con Si y Ca en vivero de palma aceitera.

\begin{tabular}{|c|c|c|c|c|c|}
\hline $\mathrm{n}$ & Producto comercial & Tratamiento & $85-135 \mathrm{dds}$ & $150-200 \mathrm{dds}$ & $\geq 215 \mathrm{dds}$ \\
\hline & & & \multicolumn{3}{|c|}{ 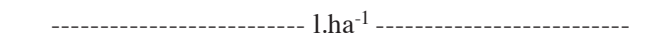 } \\
\hline 1 & Testigo & Test & --- & --- & --- \\
\hline 2 & Cal $56^{\circledR}$ & Ca56 & 0,6 & 1,05 & 1,5 \\
\hline 3 & Supa Cobre ${ }^{\circledR}$ & $\mathrm{SupCu}$ & 0,6 & 1,05 & 1,5 \\
\hline 4 & Polisulfuro de $\mathrm{Ca}^{\circledR}$ & PolCal & 0,6 & 1,05 & 1,5 \\
\hline 5 & Calcimax $^{\circledR}$ & Calmax & 0,6 & 1,05 & 1,5 \\
\hline 6 & Sifol Plus ${ }^{\circledR}$ & Sifol & 0,6 & 1,05 & 1,5 \\
\hline 7 & Alexin $^{\circledR}$ & Alexi & 0,6 & 1,05 & 1 \\
\hline 8 & Stan $\mathrm{SKH}^{\circledR}$ & Stan & 0,6 & 1,05 & 1,5 \\
\hline 9 & Firmeza ${ }^{\circledR}$ & Firmez & 0,6 & 1,05 & 1,5 \\
\hline
\end{tabular}

dds: días después de siembra.

Se realizó la calibración del equipo previo a cada aspersión, de manera que el caldo de aplicación fuera suficiente para humedecer homogéneamente toda la superficie foliar. Se utilizó una bomba de espalda con comprensor de batería modelo "Battery sprayer KB-16E-8", de 1,8 - 2,2 amperios y 3 bares de presión en trabajo. Para la calibración del equipo, se aplicó un volumen de agua sobre 4 filas bordes y se midió el remanente para obtener la cantidad total de agua de aplicación. Posteriormente, fueron asperjadas las filas con las dosis correspondientes a cada tratamiento.

\section{Variables evaluadas}

Se utilizaron profundidades de $0-15 \mathrm{~cm}$ y de $15-30 \mathrm{~cm}$ en los suelos en 2 fechas distintas, a los 130 dds y 300 dds, en bolsas para el análisis general del experimento. Se determinaron los contenidos de Acidez intercambiable con $\mathrm{KCl}$ y Al intercambiable por retitulación con $\mathrm{HCl}$ al 0,01 M, pH, Ca, Mg, K, P, Fe, Zn, Mn y Cu, mediante la solución extractora Mehlich III y Si, a través de la extracción con ácido acético según la metodología de Lobo (2013). Para las hojas lanceoladas y bifurcadas se recolectó el total de la hoja 1 en los muestreos realizados a los 175 y 220 dds. Para la hoja 1 expandidas (con separaciones entre los folios) se recolectaron 2 folios a cada lado según la metodología de Rodríguez y Jiménez (2013), recolectados a los 265 y 300 dds. Las muestras foliares se recolectaron 8 días después de la aspersión foliar, se extrajeron las venas de las hojas y se secaron a $60^{\circ} \mathrm{C}$ durante 3 días. Posteriormente, fueron enviadas al laboratorio de Suelos y Foliares de la Compañía Palma Tica S. A., donde se realizaron pruebas de presencia de Si mediante la metodología de (Lobo 2013), N por combustión seca, $\mathrm{P}, \mathrm{K}, \mathrm{Ca}, \mathrm{Mg}, \mathrm{S}, \mathrm{Fe}, \mathrm{Zn}, \mathrm{Mn}$, $\mathrm{Cu}$, a través de la digestión húmeda con $\mathrm{HNO}_{3}$ y determinación por espectrometría de Emisión atómica con Plasma (ICP). 
Se realizaron mediciones de crecimiento morfológico de la planta según la metodología de Ramírez y Muñoz (2010). Se determinó el largo de raquis en $\mathrm{cm}$ a partir de la base de la hoja 1 hasta el meristemo apical. Se midió el diámetro de bulbo que se encuentra en la base de la palma, con un vernier en $\mathrm{cm}$. Por último, se contaron las hojas totales emitidas por las plantas en un periodo de tiempo determinado.

La evaluación del Complejo de Necrosis Foliar (CNF) se realizó mediante una escala visual (medida que se desarrolla con la vista humana, método aclarado en el párrafo) representada por 5 grados de severidad en las plantas. Los grados se fundamentaron de la siguiente manera: $1=$ representó el $3 \%$ de la hoja o planta dañada, 2 $=7 \%, 3=15 \%, 4=40 \%, 5=75 \%$. Estas se realizaron para la hoja 1 y para la planta total, de manera que esta última cuantificara la severidad en fechas anteriores. Las evaluaciones de CNF se cuantificaron en conjunto con las mediciones morfológicas a $\operatorname{los} 85,130,175,220,265$ y 300 dds.

\section{Diseño experimental}

El experimento fue constituido por 9 tratamientos y 4 repeticiones con un diseño experimental de Bloques Completos al Azar. Los bloques fueron ubicados en un sector céntrico, con historial de enfermedades foliares y sin problemas de topografía. Se prepararon 4 bloques, cada bloque fue representado por 870 plantas con un tamaño de $1096 \mathrm{~m}^{2}$, cada repetición tenía un total de 30 plantas.

Para el análisis de los datos morfológicos se realizaron índices de áreas bajo la curva (abc), para reunir la información de las variables en el tiempo según la metodología de Garbanzo et al . (2018). Posteriormente, los datos fueron sometidos a un análisis de varianza, se calcularon las diferencias estadísticas mediante pruebas de Dunnett test $(\alpha=0,05)$ para los datos morfológicos, Kruskal Wallis $(\alpha=0,05)$ para las variables no paramétricas y para los análisis químicos LSD $(\alpha=0,05)$. Los cálculos fueron realizados por medio del programa estadístico Rstudio versión 0.98.1102.

\section{RESULTADOS}

Las aplicaciones de fuentes de $\mathrm{Ca}$ y $\mathrm{Si}$ foliar no mostraron un efecto significativo en la tolerancia al Complejo de Necrosis Foliar (CNF) a través del tiempo (Cuadro 4). Al analizar el índice de área bajo la curva, el Supcu mostró una tolerancia altamente significativa $(\mathrm{p}<0,001)$ en la hoja $1(808,6)$ en comparación al testigo $(1017,3)$, este mismo comportamiento mostró tendencias en la severidad de las hojas totales, mientras que Alexi $(1269,7)$ y Cal56 $(1230,1)$ mostraron la mayor severidad con significancia en comparación al modelo de referencia. Se encontró un mayor crecimiento significativo $(\mathrm{p}=0,001)$ en el largo de raquis en el Sifol $(3290,2)$ en comparación al referente $(3018,4)$. Este mismo tratamiento mostró un mayor diámetro de bulbo $(647,9)$ que el prototipo base $(606,8)$, mientras que los demás fertilizantes foliares no mostraron diferencias. El PolCal mostró un mayor número de hojas totales $(1375,1)$ notable en referencia al testigo $(1312,8)$. Por último, el índice calculado para el largo de la hoja reveló una diferencia relevante cuando se aplicó el Firmez $(803,0)$ en comparación al testigo $(763,4)$. 
Cuadro 4. Índice de área bajo la curva en crecimientos morfológico y severidad de Complejo de Necrosis Foliar en plantas de palma aceitera, tratadas con distintas fuentes de Si y Ca foliar en fase de vivero.

\begin{tabular}{|c|c|c|c|c|c|c|}
\hline \multirow{2}{*}{ Tratamientos } & $\begin{array}{c}\text { Severidad } \\
\text { Hoja } 1\end{array}$ & $\begin{array}{c}\text { Severidad } \\
\text { Total }\end{array}$ & Largo raquis & Hojas totales & $\begin{array}{c}\text { Diámetro } \\
\text { bulbo }\end{array}$ & Largo Hoja \\
\hline & & -1---- & \multicolumn{2}{|c|}{ Índices de áreas bajo la curva } & & - \\
\hline Alexi & 1269,7 & 1854,6 & 2889,4 & 1284,2 & 581,7 & 767,1 \\
\hline Ca56 & 1230,1 & 1979,3 & 2963,8 & 1360,7 & 608,5 & 780,9 \\
\hline Calmax & 1151,6 & 1829,4 & 2975,6 & 1338,7 & 597,8 & 741,3 \\
\hline Firmez & 1141,4 & 1803,6 & 2987,5 & 1294,6 & 606,0 & 803,4 \\
\hline PolCal & 1176,9 & 1821,8 & 2950,6 & 1375,1 & 613,5 & 761,7 \\
\hline Sifol & 1125,9 & 1776,2 & 3290,2 & 1359,1 & 647,9 & 780,9 \\
\hline Stan & 1204,6 & 1921,7 & 2984,2 & 1332,0 & 596,2 & 792,1 \\
\hline $\mathrm{SupCu}$ & 808,6 & 1633,5 & 3033,1 & 1333,3 & 605,2 & 760,8 \\
\hline Test & 1017,3 & 1818,2 & 3018,4 & 1312,8 & 606,8 & 763,4 \\
\hline \multicolumn{2}{|l|}{ Comparaciones } & & \multicolumn{3}{|c|}{ Dunnett test $\operatorname{Pr}(>|t|)$} & \\
\hline Alexi - test & $<0,001$ & 1,00 & 0,180 & 0,411 & 0,026 & 1,00 \\
\hline Ca56 - test & $<0,001$ & 0,07 & 0,932 & 0,032 & 1,00 & 1,00 \\
\hline Calmax - test & 0,066 & 1,00 & 0,982 & 0,521 & 0,869 & 0,449 \\
\hline Firmez - test & 0,078 & 1,00 & 0,998 & 0,848 & 1,000 & 0,022 \\
\hline PolCal - test & 0,014 & 1,00 & 0,819 & 0,001 & 0,969 & 1,00 \\
\hline Sifol - test & 0,410 & 0,989 & $<0,001$ & 0,041 & $<0,001$ & 0,698 \\
\hline Stan - test & 0,002 & 0,673 & 0,996 & 0,814 & 0,756 & 0,185 \\
\hline $\mathrm{SupCu}$ - test & $<0,001$ & 0,07 & 1,00 & 0,750 & 1,00 & 1,00 \\
\hline
\end{tabular}

n = 36288; Variables morfológicas y severidad evaluados a los 85, 127, 176, 219, 261, 304 días después de siembra. Probabilidades por debajo de $5 \%(\operatorname{Pr}(>|t|)<0,05)$ representan diferencias significativas de tratamientos en comparación al testigo.

Las fuentes de Si y Ca foliar mostraron tolerancia significativa en la severidad de CNF de la hoja 1 hasta los 220 dds (Figura 1). Al evaluar el comportamiento del porcentaje de severidad en la hoja 1, el SupCu mostró tolerancia importante $(\alpha<0,05)$ a CNF y mantiene un efecto desde los 220 dds hasta los 304 dds.
Sin embargo, en el Calcimax a los 219 no se diferencia del SupCu y los demás fertilizantes foliares. Al analizar la severidad del CNF a los 304 dds al igual que el SupCu, el Firmez mostraron mayor tolerancia a enfermedades foliares y difieren estadísticamente del resto de los tratamientos. 


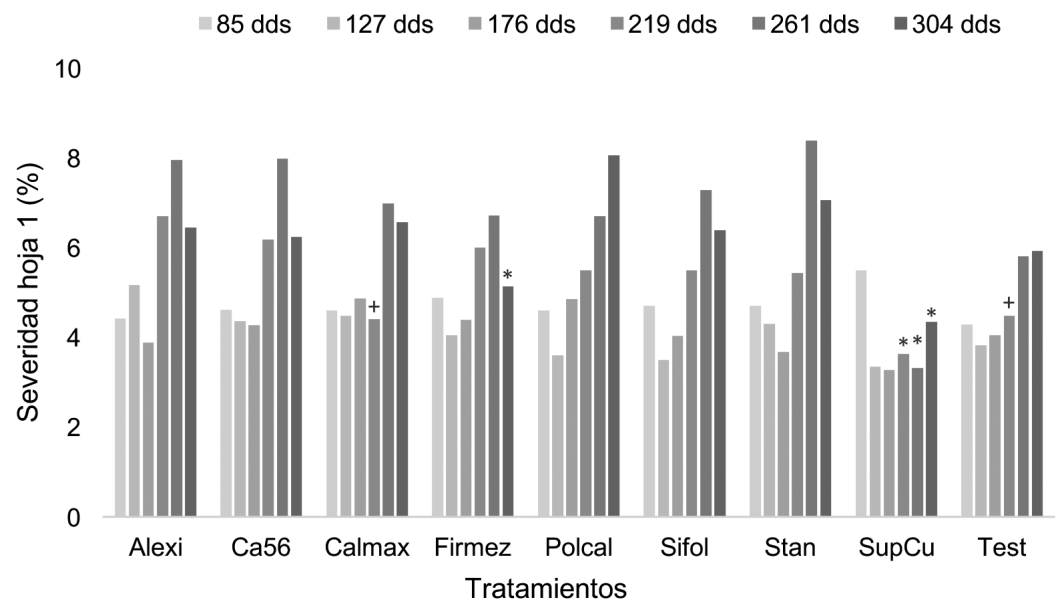

$\mathrm{n}=6048$; Severidad en hoja 1 evaluada a los 85, 127, 176, 219,261, 304 días después de siembra. * Probabilidades por debajo de $5 \%(\alpha<0,05)$ representan diferencias significativas entre tratamientos según pruebas de Kruskal Wallis. + no existen diferencias entre y el resto de tratamientos.

Fig. 1. Severidad del Complejo de Necrosis Foliar en la hoja 1 en plantas de palma aceitera entre los 85 y 304 dds, tratadas con distintas fuentes de Si y Ca foliar en fase de vivero.

Al evaluar la severidad de CNF en las hojas totales se encontró un aumento en la tolerancia a los 261 dds (Figura 2). Las plantas lograron una tolerancia significativa en el SupCu a los 261 dds y con diferencias representativas con respecto al resto de los tratamientos.
Este efecto se mantuvo en el SupCu hasta los 304 dds, sin embargo, no varió estadísticamente de Sifol y Firmez. Los demás fertilizantes foliares no mostraron diferencias notables en relación con el testigo en las distintas fechas de evaluación.

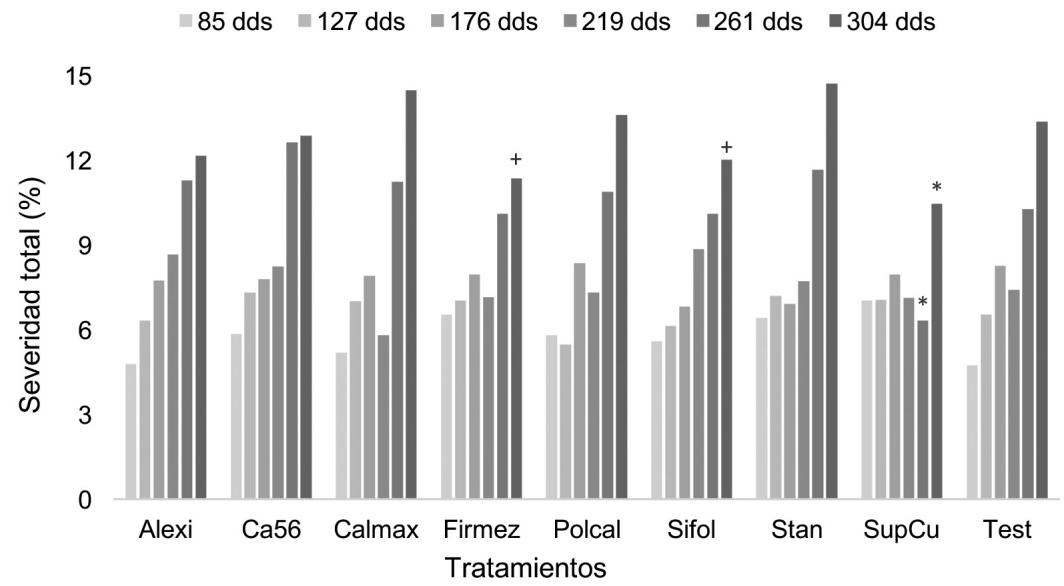

$\mathrm{n}=6048 ;$ Severidad total evaluada a los 85, 127, 176, 219, 261, 304 días después de siembra. *Probabilidades por debajo de 5\% $(\alpha<0,05)$ representan diferencias significativas entre tratamientos según pruebas de Kruskal Wallis. + no existen diferencias entre y el resto de tratamientos.

Fig. 2. Severidad de Complejo de Necrosis Foliar en las hojas totales en plantas de palma aceitera entre los 85 y 304 dds, tratadas con distintas fuentes de Si y Ca foliar en fase de vivero.

Agronomía Costarricense 42(2): 43-57. ISSN:0377-9424 / 2018 
Las plantas mostraron un mayor crecimiento en el diámetro de bulbo cuando se aplicaron fuentes de Si y Ca foliar a partir de los 127 dds (Figura 3). El efecto solo se logró de forma destacada en el Sifol a partir de los 127 dds y se mantuvo hasta los $261 \mathrm{dds}$. Sin embargo, el
PolCal no se diferenció estadísticamente del Sifol a los 127 dds y de Firmez y SupCu a los 176 dds. Los demás tratamientos no mostraron diferencias mínimas significativas entre ellos y las fechas de evaluación.

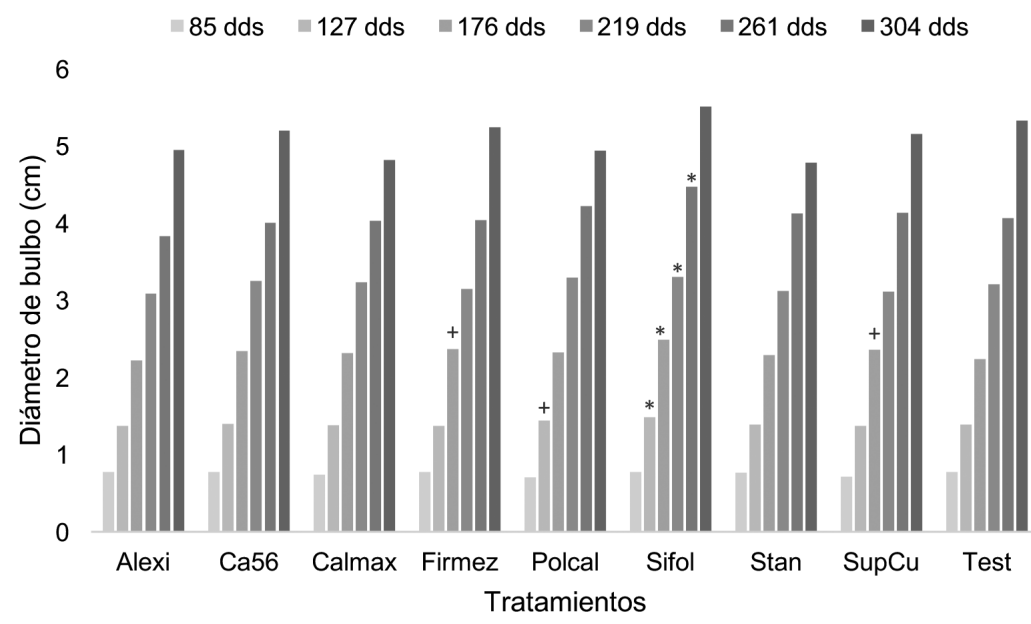

n = 6048; Diámetro de bulbo evaluado a los 85, 127, 176, 219, 261, 304 días después de siembra. * Probabilidades por debajo de $5 \%(\alpha<0,05)$ representan diferencias significativas entre tratamientos según pruebas de Kruskal Wallis. + no existen diferencias entre y el resto de tratamientos.

Fig. 3. Diámetro de bulbo en plantas de palma aceitera entre los 85 y 304 dds, tratadas con distintas fuentes de Si y Ca foliar en fase de vivero.

No hubo diferencias significativas en la concentración de macronutrimentos en la hoja 1 de las plántulas con la aplicación de fertilizantes foliares (Cuadro 5). Para N, varió entre $3,25 \%$ y $3,92 \%$ a los 175 y 310 dds y no mostró diferencia estadística $(\alpha<0,05)$ entre los tratamientos. El P varió entre $0,20 \% \mathrm{y}$ $0,26 \%$ en todas las fechas de muestreo. Para K en plantas, no mostró diferencias significativas entre los fertilizantes foliares, con una variación entre 1,23 y 1,65\%. El contenido de $\mathrm{Ca}$ no mostró diferencias estadísticas, sin embargo, el SupCu mostró una tendencia de mayor concentración en la última fecha. El Ca en las plantas de vivero varió entre un 0,79 y $1,06 \%$ en la hoja 1. El Mg en la planta fue mayor a los 175 dds, no obstante, después de los 265 dds, la concentración de $\mathrm{Mg}$ no mostró diferencia, por lo que osciló entre 0,25 y $0,35 \%$. La concentración de $\mathrm{S}$ se encontró entre 0,23 y $0,31 \%$, donde no mostró diferencias estadísticamente significativas a los 175 y 265 dds, sin embargo, a los 310 dds mostró tendencia de mayor concentración en SupCU $(0,31)$, por lo que se encontraron diferencias estadísticas en comparación al Calmax $(0,27 \%)$. 
Cuadro 5. Efecto de las fuentes de Si y Ca foliar en la concentración de N, P, K, Ca, Mg y S en hoja 1 de palma aceitera.

\begin{tabular}{|c|c|c|c|c|c|c|}
\hline \multirow{2}{*}{ Tratamientos } & $\mathrm{N}$ & $\mathrm{P}$ & $\mathrm{K}$ & $\mathrm{Ca}$ & $\mathrm{Mg}$ & $\mathrm{S}$ \\
\hline & \multicolumn{6}{|c|}{ 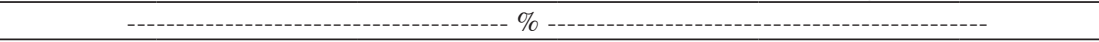 } \\
\hline & \multicolumn{6}{|c|}{ 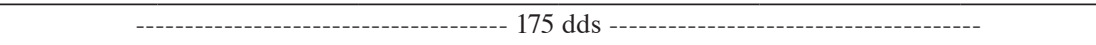 } \\
\hline Alexi & $3,49 a^{*}$ & $0,25 \mathrm{a}$ & $1,59 \mathrm{a}$ & 1,06 a & $0,33 \mathrm{ab}$ & $0,31 \mathrm{a}$ \\
\hline Ca56 & 3,49 a & $0,25 \mathrm{a}$ & $1,57 \mathrm{a}$ & $0,97 \mathrm{a}$ & $0,31 \mathrm{~b}$ & $0,28 \mathrm{a}$ \\
\hline Calmax & $3,41 \mathrm{a}$ & $0,26 \mathrm{a}$ & $1,55 \mathrm{a}$ & $1,03 \mathrm{a}$ & $0,31 \mathrm{~b}$ & $0,29 \mathrm{a}$ \\
\hline Firmez & $3,25 \mathrm{a}$ & $0,25 \mathrm{a}$ & $1,54 \mathrm{a}$ & $0,98 \mathrm{a}$ & $0,31 \mathrm{~b}$ & $0,28 \mathrm{a}$ \\
\hline PolCal & $3,45 \mathrm{a}$ & $0,25 \mathrm{a}$ & $1,56 \mathrm{a}$ & $1,00 \mathrm{a}$ & $0,31 \mathrm{~b}$ & $0,27 \mathrm{a}$ \\
\hline Sifol & $3,48 \mathrm{a}$ & $0,25 \mathrm{a}$ & $1,54 \mathrm{a}$ & $1,01 \mathrm{a}$ & $0,32 \mathrm{~b}$ & $0,28 \mathrm{a}$ \\
\hline Stan & $3,28 \mathrm{a}$ & $0,25 \mathrm{a}$ & $1,65 \mathrm{a}$ & $1,00 \mathrm{a}$ & $0,32 \mathrm{~b}$ & $0,30 \mathrm{a}$ \\
\hline $\mathrm{SupCu}$ & $3,49 \mathrm{a}$ & $0,26 \mathrm{a}$ & $1,63 \mathrm{a}$ & $1,00 \mathrm{a}$ & $0,32 \mathrm{~b}$ & $0,29 \mathrm{a}$ \\
\hline Test & $3,35 \mathrm{a}$ & $0,24 \mathrm{a}$ & $1,45 \mathrm{a}$ & $1,00 \mathrm{a}$ & $0,35 \mathrm{a}$ & $0,29 \mathrm{a}$ \\
\hline \multirow[t]{2}{*}{$\operatorname{Adj~r~}^{2}$} & 0,48 & 0,38 & 0,40 & 0,28 & 0,64 & 0,42 \\
\hline & \multicolumn{6}{|c|}{ 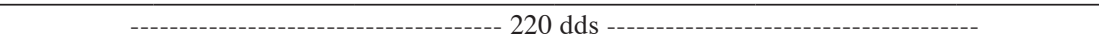 } \\
\hline Alexi & $3,84 \mathrm{a}$ & $0,20 \mathrm{c}$ & $1,30 \mathrm{a}$ & $0,91 \mathrm{abc}$ & $0,26 \mathrm{ab}$ & $0,27 \mathrm{ab}$ \\
\hline Ca56 & $3,75 \mathrm{a}$ & $0,20 \mathrm{bc}$ & $1,23 \mathrm{a}$ & $0,87 \mathrm{bc}$ & $0,25 \mathrm{~b}$ & $0,23 \mathrm{ab}$ \\
\hline Calmax & $3,92 \mathrm{a}$ & $0,20 \mathrm{c}$ & $1,27 \mathrm{a}$ & $0,90 \mathrm{abc}$ & $0,25 \mathrm{ab}$ & $0,27 \mathrm{ab}$ \\
\hline Firmez & $3,82 \mathrm{a}$ & $0,22 \mathrm{a}$ & $1,29 \mathrm{a}$ & $0,89 \mathrm{abc}$ & $0,26 \mathrm{ab}$ & $0,29 \mathrm{a}$ \\
\hline PolCal & $3,81 \mathrm{a}$ & $0,21 \mathrm{bc}$ & $1,31 \mathrm{a}$ & $0,84 \mathrm{c}$ & $0,25 \mathrm{~b}$ & $0,25 \mathrm{bc}$ \\
\hline Sifol & $3,82 \mathrm{a}$ & $0,19 \mathrm{c}$ & $1,25 \mathrm{a}$ & $0,98 \mathrm{a}$ & $0,27 \mathrm{ab}$ & $0,28 \mathrm{ab}$ \\
\hline Stan & $3,64 \mathrm{a}$ & $0,19 \mathrm{c}$ & $1,37 \mathrm{a}$ & $0,88 \mathrm{abc}$ & $0,26 \mathrm{ab}$ & $0,27 \mathrm{ab}$ \\
\hline $\mathrm{SupCu}$ & $3,73 \mathrm{a}$ & $0,20 \mathrm{bc}$ & $1,36 \mathrm{a}$ & $0,97 \mathrm{ab}$ & $0,28 \mathrm{a}$ & $0,25 \mathrm{bc}$ \\
\hline Test & $3,60 \mathrm{a}$ & $0,22 \mathrm{ab}$ & $1,31 \mathrm{a}$ & $0,94 \mathrm{abc}$ & $0,27 \mathrm{ab}$ & $0,29 \mathrm{a}$ \\
\hline \multirow[t]{2}{*}{$\operatorname{Adj~r~}^{2}$} & 0,60 & 0,78 & 0,71 & 0,75 & 0,74 & 0,01 \\
\hline & \multicolumn{6}{|c|}{--------------------------------------- 265 dds -------------------------------------- } \\
\hline Alexi & 3,36 a & $0,22 \mathrm{a}$ & $1,58 \mathrm{a}$ & 0,86 a & $0,25 \mathrm{a}$ & $0,26 \mathrm{a}$ \\
\hline Ca56 & 3,49 a & $0,23 \mathrm{a}$ & $1,57 \mathrm{a}$ & $0,93 \mathrm{a}$ & $0,27 \mathrm{a}$ & $0,28 \mathrm{a}$ \\
\hline Calmax & $3,26 \mathrm{a}$ & $0,22 \mathrm{a}$ & $1,58 \mathrm{a}$ & $0,90 \mathrm{a}$ & $0,26 \mathrm{a}$ & $0,27 \mathrm{a}$ \\
\hline Firmez & $3,38 \mathrm{a}$ & $0,22 \mathrm{a}$ & $1,48 \mathrm{a}$ & $0,91 \mathrm{a}$ & $0,26 \mathrm{a}$ & $0,27 \mathrm{a}$ \\
\hline PolCal & $3,33 \mathrm{a}$ & $0,23 \mathrm{a}$ & $1,62 \mathrm{a}$ & $0,85 \mathrm{a}$ & $0,25 \mathrm{a}$ & $0,27 \mathrm{a}$ \\
\hline Sifol & $3,29 \mathrm{a}$ & $0,23 \mathrm{a}$ & $1,63 \mathrm{a}$ & $0,87 \mathrm{a}$ & $0,25 \mathrm{a}$ & $0,27 \mathrm{a}$ \\
\hline Stan & $3,42 \mathrm{a}$ & $0,22 \mathrm{a}$ & $1,58 \mathrm{a}$ & $0,87 \mathrm{a}$ & $0,25 \mathrm{a}$ & $0,25 \mathrm{a}$ \\
\hline $\mathrm{SupCu}$ & $3,54 \mathrm{a}$ & $0,22 \mathrm{a}$ & $1,54 \mathrm{a}$ & $0,90 \mathrm{a}$ & $0,26 \mathrm{a}$ & $0,27 \mathrm{a}$ \\
\hline Test & $3,50 \mathrm{a}$ & $0,22 \mathrm{a}$ & $1,59 \mathrm{a}$ & $0,84 \mathrm{a}$ & $0,25 \mathrm{a}$ & $0,27 \mathrm{a}$ \\
\hline \multirow[t]{2}{*}{$\operatorname{Adj~r~}^{2}$} & 0,47 & 0,42 & 0,28 & 0,24 & 0,33 & 0,45 \\
\hline & \multicolumn{6}{|c|}{ 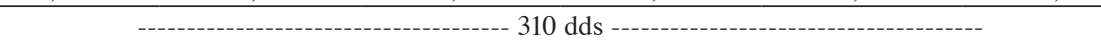 } \\
\hline Alexi & $3,62 \mathrm{a}$ & $0,21 \mathrm{a}$ & $1,46 \mathrm{a}$ & $0,91 \mathrm{a}$ & $0,27 \mathrm{a}$ & $0,29 \mathrm{ab}$ \\
\hline Ca56 & $3,43 \mathrm{a}$ & $0,22 \mathrm{a}$ & $1,62 \mathrm{a}$ & $0,79 \mathrm{a}$ & $0,25 \mathrm{a}$ & $0,28 \mathrm{ab}$ \\
\hline Calmax & $3,41 \mathrm{a}$ & $0,21 \mathrm{a}$ & $1,53 \mathrm{a}$ & $0,79 \mathrm{a}$ & $0,25 \mathrm{a}$ & $0,27 \mathrm{~b}$ \\
\hline Firmez & $3,48 \mathrm{a}$ & $0,22 \mathrm{a}$ & $1,62 \mathrm{a}$ & $0,83 \mathrm{a}$ & $0,27 \mathrm{a}$ & $0,30 \mathrm{ab}$ \\
\hline PolCal & $3,46 \mathrm{a}$ & $0,22 \mathrm{a}$ & $1,60 \mathrm{a}$ & $0,79 \mathrm{a}$ & $0,24 \mathrm{a}$ & $0,28 \mathrm{ab}$ \\
\hline Sifol & $3,39 \mathrm{a}$ & $0,21 \mathrm{a}$ & $1,53 \mathrm{a}$ & $0,80 \mathrm{a}$ & $0,25 \mathrm{a}$ & $0,27 \mathrm{ab}$ \\
\hline Stan & $3,42 \mathrm{a}$ & $0,21 \mathrm{a}$ & $1,51 \mathrm{a}$ & $0,83 \mathrm{a}$ & $0,25 \mathrm{a}$ & $0,29 \mathrm{ab}$ \\
\hline $\mathrm{SupCu}$ & $3,67 \mathrm{a}$ & $0,22 \mathrm{a}$ & $1,49 \mathrm{a}$ & $0,92 \mathrm{a}$ & $0,29 \mathrm{a}$ & $0,31 \mathrm{a}$ \\
\hline Test & $3,37 \mathrm{a}$ & $0,21 \mathrm{a}$ & $1,56 \mathrm{a}$ & $0,85 \mathrm{a}$ & $0,27 \mathrm{a}$ & $0,30 \mathrm{ab}$ \\
\hline $\operatorname{Adj~} \mathrm{r}^{2}$ & 0,47 & 0,50 & 0,36 & 0,36 & 0,48 & 0,64 \\
\hline
\end{tabular}

$\mathrm{n}=864$; Severidad en hoja 1 evaluada a los 85, 127, 176, 219, 261, 304 días después de siembra. * Letras distintas denotan diferencia significativa de acuerdo con prueba LSD Test (p.adj= bonferroni, $\alpha<0,05)$ del modelo lineal (LM). 
El contenido foliar de Si en la hoja 1 en las palmas, no aumentó con las aplicaciones foliares sin embargo, aumentó el contenido de $\mathrm{Cu}$ y $\mathrm{B}$ en algunas fuentes (Cuadro 6). Al evaluar la concentración de Si foliar a los 175, 220, 265 y 310 dds no se encontró una diferencia significativa $(\alpha<0,05)$ entre los tratamientos, pero mostró tendencias de mayor acumulación en Alexi $\left(0,94 \mathrm{mg} \cdot \mathrm{kg}^{-1}\right)$ y $\mathrm{SupCu}\left(1 \mathrm{mg} \cdot \mathrm{kg}^{-1}\right)$ a los 310 dds. Las concentraciones de $\mathrm{Cu}$ en las plantas mostraron diferencias mínimas significativas en todas las fechas de muestreo; se encontró más contenido en $\mathrm{SupCu}(32,1-$ $\left.52,9 \mathrm{mg} \cdot \mathrm{kg}^{-1}\right)$ y Sifol $\left(16,3-26,1 \mathrm{mg} \cdot \mathrm{kg}^{-1}\right)$ en comparación con el resto de los fertilizantes foliares $\left(8,9-12,7 \mathrm{mg} \cdot \mathrm{kg}^{-1}\right)$. 
Cuadro 6. Efecto de la aplicación foliar de fuentes de Si y Ca en la concentración de Si, Al, Fe, Cu, Zn, Mn y B en hoja 1 de palma aceitera.

\begin{tabular}{|c|c|c|c|c|c|c|c|}
\hline \multirow{2}{*}{ Tratamientos } & $\mathrm{Si}$ & $\mathrm{Al}$ & $\mathrm{Fe}$ & $\mathrm{Cu}$ & $\mathrm{Zn}$ & Mn & $\mathrm{B}$ \\
\hline & $\%$ & \multicolumn{6}{|c|}{ 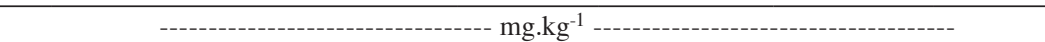 } \\
\hline & \multicolumn{7}{|c|}{ 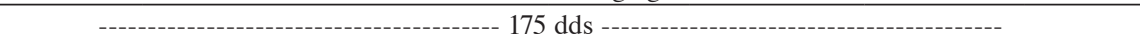 } \\
\hline Alexi & $1,13 \mathrm{a}$ & $180,36 \mathrm{a}$ & 213,59 a & $11,44 \mathrm{c}$ & $16,71 \mathrm{a}$ & $126,61 \mathrm{a}$ & $19,93 \mathrm{~b}$ \\
\hline Ca56 & $0,90 \mathrm{a}$ & $219,63 \mathrm{a}$ & 230,26 a & $11,94 \mathrm{c}$ & $18,83 \mathrm{a}$ & $109,29 \mathrm{a}$ & $17,31 \mathrm{ab}$ \\
\hline Calmax & $0,96 \mathrm{a}$ & $164,84 \mathrm{a}$ & $193,22 \mathrm{a}$ & $11,50 \mathrm{c}$ & $17,47 \mathrm{a}$ & $117,88 \mathrm{a}$ & $16,69 \mathrm{ab}$ \\
\hline Firmez & $1,17 \mathrm{a}$ & 248,72 a & $252,03 \mathrm{a}$ & $12,75 \mathrm{c}$ & $17,33 \mathrm{a}$ & $101,37 \mathrm{a}$ & $15,92 \mathrm{ab}$ \\
\hline PolCal & $1,00 \mathrm{a}$ & $150,63 \mathrm{a}$ & $177,79 \mathrm{a}$ & $12,15 \mathrm{c}$ & $17,07 \mathrm{a}$ & $108,11 \mathrm{a}$ & $14,80 \mathrm{ab}$ \\
\hline Sifol & $0,96 \mathrm{a}$ & $175,96 \mathrm{a}$ & $213,10 \mathrm{a}$ & $26,07 \mathrm{~b}$ & $16,72 \mathrm{a}$ & $111,33 \mathrm{a}$ & $17,46 \mathrm{ab}$ \\
\hline Stan & $1,04 \mathrm{a}$ & $251,98 \mathrm{a}$ & 261,21 a & $12,28 \mathrm{c}$ & 16,67 a & 114,49 a & $19,94 \mathrm{~b}$ \\
\hline $\mathrm{SupCu}$ & $0,94 \mathrm{a}$ & $181,01 \mathrm{a}$ & $190,89 \mathrm{a}$ & $51,71 \mathrm{a}$ & $19,02 \mathrm{a}$ & $105,12 \mathrm{a}$ & $18,09 \mathrm{ab}$ \\
\hline Test & $1,22 \mathrm{a}$ & $191,99 \mathrm{a}$ & $227,78 \mathrm{a}$ & $9,38 \mathrm{c}$ & $15,84 \mathrm{a}$ & $132,40 \mathrm{a}$ & $13,28 \mathrm{a}$ \\
\hline \multirow[t]{2}{*}{$\operatorname{Adj~r}^{2}$} & 0,57 & 0,49 & 0,53 & 0,99 & 0,52 & 0,59 & 0,56 \\
\hline & & \multicolumn{6}{|c|}{ 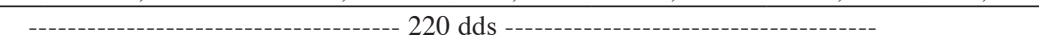 } \\
\hline Alexi & $0,97 \mathrm{~b}$ & $31,97 \mathrm{a}$ & $92,75 \mathrm{a}$ & $9,82 \mathrm{c}$ & $14,80 \mathrm{bc}$ & $112,56 \mathrm{a}$ & $24,48 \mathrm{~b}$ \\
\hline Ca56 & $1,03 \mathrm{ab}$ & $35,05 \mathrm{a}$ & $95,83 \mathrm{a}$ & $10,52 \mathrm{c}$ & $16,88 \mathrm{abc}$ & $111,32 \mathrm{a}$ & $22,31 \mathrm{~b}$ \\
\hline Calmax & $1,05 \mathrm{ab}$ & $52,01 \mathrm{a}$ & $109,78 \mathrm{a}$ & $9,90 \mathrm{c}$ & $15,34 \mathrm{bc}$ & $115,05 \mathrm{a}$ & $23,76 \mathrm{~b}$ \\
\hline Firmez & $1,13 \mathrm{ab}$ & 43,16 a & $105,74 \mathrm{a}$ & $11,07 \mathrm{c}$ & $18,53 \mathrm{a}$ & $109,15 \mathrm{a}$ & $24,25 \mathrm{~b}$ \\
\hline PolCal & $1,00 \mathrm{~b}$ & $27,42 \mathrm{a}$ & 87,44 a & $10,65 \mathrm{c}$ & $15,73 \mathrm{abc}$ & $107,25 \mathrm{a}$ & $22,21 \mathrm{~b}$ \\
\hline Sifol & $1,15 \mathrm{ab}$ & $47,51 \mathrm{a}$ & $108,83 \mathrm{a}$ & $22,41 \mathrm{~b}$ & $15,26 \mathrm{bc}$ & $135,86 \mathrm{a}$ & $26,70 \mathrm{ab}$ \\
\hline Stan & $1,04 \mathrm{ab}$ & $39,34 \mathrm{a}$ & $94,66 \mathrm{a}$ & $10,06 \mathrm{c}$ & $14,32 \mathrm{c}$ & $112,97 \mathrm{a}$ & $21,84 \mathrm{~b}$ \\
\hline $\mathrm{SupCu}$ & $1,07 \mathrm{ab}$ & $30,95 \mathrm{a}$ & 97,81 a & $52,90 \mathrm{a}$ & $16,76 \mathrm{abc}$ & $118,35 \mathrm{a}$ & $26,22 \mathrm{ab}$ \\
\hline Test & $1,26 \mathrm{a}$ & $33,08 \mathrm{a}$ & $98,92 \mathrm{a}$ & $10,16 \mathrm{c}$ & $17,40 \mathrm{ab}$ & $129,89 \mathrm{a}$ & $30,84 \mathrm{a}$ \\
\hline \multirow[t]{2}{*}{ Adj $r^{2}$} & 0,54 & 0,55 & 0,90 & 0,80 & 0,44 & 0,64 & 0,59 \\
\hline & & \multicolumn{6}{|c|}{ - } \\
\hline Alexi & $0,76 \mathrm{a}$ & $22,26 \mathrm{a}$ & $75,80 \mathrm{a}$ & $10,38 \mathrm{~b}$ & $16,35 \mathrm{a}$ & $99,63 \mathrm{a}$ & $25,22 \mathrm{~b}$ \\
\hline Ca56 & $0,81 \mathrm{a}$ & $24,63 \mathrm{a}$ & $72,87 \mathrm{a}$ & $11,01 \mathrm{~b}$ & $16,85 \mathrm{a}$ & $109,68 \mathrm{a}$ & $27,09 \mathrm{~b}$ \\
\hline Calmax & $0,82 \mathrm{a}$ & $22,80 \mathrm{a}$ & 70,68 a & $10,86 \mathrm{~b}$ & $17,16 \mathrm{a}$ & $103,92 \mathrm{a}$ & $26,51 \mathrm{~b}$ \\
\hline Firmez & $0,83 \mathrm{a}$ & $25,48 \mathrm{a}$ & $76,84 \mathrm{a}$ & $10,98 \mathrm{~b}$ & $17,48 \mathrm{a}$ & 99,18 a & $24,50 \mathrm{~b}$ \\
\hline PolCal & $0,76 \mathrm{a}$ & 19,48 a & $66,69 \mathrm{a}$ & $11,42 \mathrm{~b}$ & $17,90 \mathrm{a}$ & $96,42 \mathrm{a}$ & $23,83 \mathrm{~b}$ \\
\hline Sifol & $0,80 \mathrm{a}$ & $28,97 \mathrm{a}$ & 77,92 a & $16,28 \mathrm{~b}$ & $16,97 \mathrm{a}$ & $106,33 \mathrm{a}$ & $24,94 \mathrm{~b}$ \\
\hline Stan & $0,85 \mathrm{a}$ & $23,46 \mathrm{a}$ & $72,72 \mathrm{a}$ & $10,97 \mathrm{~b}$ & $16,33 \mathrm{a}$ & $104,20 \mathrm{a}$ & $25,93 \mathrm{~b}$ \\
\hline $\mathrm{SupCu}$ & $0,82 \mathrm{a}$ & $29,85 \mathrm{a}$ & $79,12 \mathrm{a}$ & $50,54 \mathrm{a}$ & $18,40 \mathrm{a}$ & $113,54 \mathrm{a}$ & $27,90 \mathrm{~b}$ \\
\hline Test & $0,80 \mathrm{a}$ & 19,94 a & $70,80 \mathrm{a}$ & $10,88 \mathrm{~b}$ & $17,01 \mathrm{a}$ & $106,85 \mathrm{a}$ & $40,78 \mathrm{a}$ \\
\hline \multirow[t]{2}{*}{$\operatorname{Adj~r}^{2}$} & 0,13 & 0,30 & 0,37 & 0,91 & 0,30 & 0,26 & 0,69 \\
\hline & & \multicolumn{6}{|c|}{ 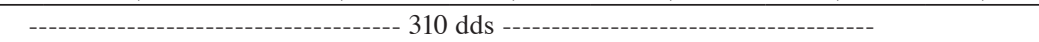 } \\
\hline Alexi & $0,94 \mathrm{a}$ & 43,67 a & 99,01 a & 8,92 a & 18,59 a & $125,94 \mathrm{a}$ & $47,17 \mathrm{ab}$ \\
\hline Ca56 & $0,79 \mathrm{a}$ & $38,05 \mathrm{a}$ & 86,05 a & $9,55 \mathrm{a}$ & $17,11 \mathrm{a}$ & 106,09 a & $36,51 \mathrm{~b}$ \\
\hline Calmax & $0,77 \mathrm{a}$ & $36,66 \mathrm{a}$ & 84,49 a & $8,98 \mathrm{a}$ & $16,98 \mathrm{a}$ & $102,85 \mathrm{a}$ & $38,38 \mathrm{ab}$ \\
\hline Firmez & $0,82 \mathrm{a}$ & $31,78 \mathrm{a}$ & 87,12 a & $9,72 \mathrm{a}$ & $16,94 \mathrm{a}$ & $102,41 \mathrm{a}$ & $37,45 \mathrm{ab}$ \\
\hline PolCal & $0,75 \mathrm{a}$ & $41,11 \mathrm{a}$ & $104,80 \mathrm{a}$ & $9,55 \mathrm{a}$ & $15,65 \mathrm{a}$ & $100,91 \mathrm{a}$ & $33,53 \mathrm{~b}$ \\
\hline Sifol & $0,73 \mathrm{a}$ & $34,93 \mathrm{a}$ & 89,62 a & $22,08 \mathrm{~b}$ & $17,19 \mathrm{a}$ & $105,47 \mathrm{a}$ & $38,25 \mathrm{ab}$ \\
\hline Stan & $0,87 \mathrm{a}$ & $33,60 \mathrm{a}$ & 86,48 a & $9,35 \mathrm{a}$ & $16,17 \mathrm{a}$ & $108,70 \mathrm{a}$ & $42,67 \mathrm{ab}$ \\
\hline $\mathrm{SupCu}$ & $1,00 \mathrm{a}$ & $40,73 \mathrm{a}$ & $95,65 \mathrm{a}$ & $32,06 \mathrm{~b}$ & $18,95 \mathrm{a}$ & $138,39 \mathrm{a}$ & $50,50 \mathrm{ab}$ \\
\hline Test & $0,83 \mathrm{a}$ & $42,89 \mathrm{a}$ & $93,34 \mathrm{a}$ & $9,16 \mathrm{a}$ & $17,59 \mathrm{a}$ & $116,44 \mathrm{a}$ & $55,89 \mathrm{a}$ \\
\hline $\operatorname{Adj~r}^{2}$ & 0,37 & 0,53 & 0,42 & 0,82 & 0,56 & 0,48 & 0,61 \\
\hline
\end{tabular}

$\mathrm{n}=1008$; Severidad en hoja 1 evaluada a los 85, 127, 176, 219, 261, 304 días después de siembra. *Letras distintas denotan diferencia significativa de acuerdo con prueba LSD Test (p.adj= bonferroni, $\alpha<0,05)$ del modelo lineal (LM). 


\section{DISCUSIÓN}

Las aplicaciones de Ca y Si foliar en palma aceitera no tuvieron efecto con la incidencia del Complejo de Necrosis Foliar en plantas de palma aceitera en vivero. Al analizar que el SupCu solo mostró una mayor tolerancia significativa en la hoja 1. La aplicación de estos nutrimentos en las hojas presentó poca penetración en la cutícula, comportamiento que concuerda con Fregoni (1986), Malavolta (1986), Trinidad y Aguilar (1999), quienes encontraron que aplicaciones de fuentes de Ca foliar penetraron solo un $50 \%$ en la cutícula después de 96 horas, la cual es muy baja en comparación a N, K, Zn, Na y Cl. Además, se sumaría el efecto de la forma de absorción de los nutrimentos, en particular de $\mathrm{Si}$, el cual se absorbe como ácido ortosílisico en forma pasiva (Ma 2015, Datnoff et al. 2007, Marschner y Marschner 2012, Pilon et al. 2014), mientras que las fuentes foliares se mostraron en forma de óxidos de silicio soluble. Por otro lado, es probable que el efecto de las constantes precipitaciones en la zona lavó el producto, sobre todo en épocas lluviosas (agosto - diciembre) y también en verano (diciembre - abril) por el riego por aspersión que se utilizó en el vivero.

La concentración de $\mathrm{Ca}$ y $\mathrm{Si}$ en el suelo fue alta y probablemente afectó los resultados del análisis foliar para ambos nutrimentos. Debido al alto contenido de $\mathrm{Ca}$ y $\mathrm{Si}$ en el suelo, la planta tendría cantidades significativas disponibles, ya que muchos se absorben en su mayoría por el flujo de masas e intercepción desde el suelo y ambos son elementos no móviles (Lambers et al. 1998, Sposito 2008, Marafon y Endres 2013, Pilon et al. 2014, Ma 2015). Es probable que parte del Si disponible para las plantas a través del tiempo, (Zapata 2004, Quero 2008, Czeschlik 2005), haya sido liberado de las estructuras que poseen las arcillas (Alvarado 1985, Klein y Hurlbut 2003, Lizcano et al. 2006, Jaramillo 2011, Alvarado et al. 2014). Se presume que el Si y Ca se absorbiera muy poco, debido a su inmovilidad y que la hoja 1 presentó importante actividad fisiológica, lo cual le permitiría la acumulación del Si en particular por la corriente transpiratoria (Yoshida 1965, Ma y Yamaji 2006), lo que explicaría que la absorción de Si foliar no es significativa en relación con la absorción por las raíces.

Al analizar las concentraciones de los nutrimentos en las hojas, solo el Cu mostró relación con la severidad del CNF. Se identificó una relación inversamente proporcional, es decir, a mayor concentración de $\mathrm{Cu}$, menor severidad de CNF en la hoja 1 y una tendencia de menor severidad en las hojas totales. Al igual que el $\mathrm{SupCu}$, el Sifol también mostró una mayor acumulación de $\mathrm{Cu}$ en la lámina foliar. Esto se debe probablemente a que ambos productos presentan $\mathrm{Cu}$ en la solución, el SupCu tiene 5,6\% ( $\left.\mathrm{p} \mathrm{v}^{-1}\right)$ de $\mathrm{Cu}$, mientras que el Sifol posee un 1,3\%. Las demás plantas no presentaron contenidos altos en las hojas, debido a que fueron obtenidos de los suelos de vivero $\left(16,3 \mathrm{mg} . \mathrm{l}^{-1}\right)$. Por su parte, el efecto del cobre sobre el control de las enfermedades está bien demostrado en otros cultivos (Albrigo y Timmer 1997, Datnoff et al. 2007, Behlay et al. 2009).

En relación con el crecimiento, el tratamiento Sifol mostró el mayor vigor de planta, cuando se analizó el diámetro de bulbo y largo de raquis al compararlo con el testigo. A diferencia del testigo y los demás fertilizantes foliares, este producto posee $\mathrm{K}$ y $\mathrm{P}$, este último en forma de fosfitos, lo cual es muy probable que mejorara los balances nutricionales de la planta y en consecuencia mayor crecimiento, más aún cuando este nutrimento se encuentra involucrado en múltiples procesos fisiológicos y de energía en las plantas (Marschner y Marschner 2012, Marafon y Endres 2013, Salim et al. 2014). Es probable que al haber concentraciones altas de $\mathrm{Cu}$, se optimizaran los balances nutricionales en la planta, lo cual mejora su crecimiento. Efectos similares encontraron Sidhu et al. (2001), donde aplicaciones de $\mathrm{Cu}$ foliar ayudaron en el crecimiento y vigor del vivero sembrado con suelo del orden Espodozol, aspecto que además coincide con el SupCu en algunas fechas durante la medición del diámetro de bulbo en comparación al Sifol. Es importante destacar que las concentraciones de muchos nutrimentos en la hoja 1 no mostraron 
diferencias significativas, sin embargo, al tener un vigor superior a los demás tratamientos, es muy probable que la acumulación de la mayoría de nutrimentos sea superior en Sifol y $\mathrm{SupCu}$ al resto de los fertilizantes foliares.

\section{CONCLUSIONES}

La concentración de $\mathrm{Si}$ y $\mathrm{Ca}$ en la hoja 1 en vivero de palma aceitera no varió con la aplicación de las fuentes foliares y no presentaron ningún efecto en el control del Complejo de Necrosis Foliar. La aplicación de productos altos en $\mathrm{Cu}$ mostraron un aumento significativo en la tolerancia de CNF en vivero de palma aceitera. Las aplicaciones de productos de $\mathrm{K}$ y $\mathrm{P}$ en forma de fosfitos aumentaron el vigor de la planta en vivero, pero no tuvo efecto en la tolerancia a CNF.

\section{AGRADECIMIENTOS}

Por el financiamiento de la investigación al Departamento de Investigación y Desarrollo de la Compañía Palma Tica S.A. Escuela de Agronomía y al Centro de Investigaciones Agronómicas de la Universidad de Costa Rica.

\section{LITERATURA CITADA}

Aguirre, C; Chávez, T; Garcia, P; Raya, J. 2007. El silicio en los organismos vivos. Revista Interciencia 32(8):504-509.

Ahmed, M; Asif, M; Goyal, A. 2012. Silicon the NonEssential Benedicial Plant Nutrient to enhanced drought tolerance in wheat. Crop Plant. University Rawalpindi - University of Alberta, Edmonton, Ab. Pakistan - Canada. 240 p.

Albrigo, L; Timmer, L. 1997. Copper fungicides - Residues for disease control and potential for spray burn. Proc. Fla. State Hort. Soc. 110:67-70.

Alvarado, A. 1985. El origen de los suelos. Centro Agronómico Tropical de Investigación y Enseñanza. Turrialba, Costa Rica. Serie de Materiales de Enseñanza $N^{\circ}$ $24.54 \mathrm{p}$.

Alvarado, A; Mata, R; Chinchilla, M. 2014. Arcillas identificadas en suelos de Costa Rica a nivel generalizado durante el período 1931-2012: I Historia, metodología de análisis y mineralogía de arcillas en suelos derivados de cenizas volcánicas. Agronomía Costarricense 38(1):75-106.
Anderson, J. 2012. Efecto a través del tiempo del uso de fertilizantes silicatados para la reducción del mal de panamá (Fusarium oxysporum f. sp. cubense) en el cultivar Gros Michel (Musa AAA). Tesis Lic. Turrialba, Costa Rica, Universidad de Costa Rica, 104 p.

Arauz, F. 2011. Fitopatología un enfoque agroecológico. 2 ed San José, Costa Rica, Editorial UCR. 514 p.

Barros, F; Sagata, E; Ferreira, L; Juliatti, F. 2010. Inducao de resistencia em plantas contra fitopatógenos. Biosci. J., Uberlandia 26(2):231-239.

Behlay, F; Belasque, J; Graham, J; Leite, R. 2009. Effect of frequency of copper applications on control of citrus canker and the yield of young bearing sweet orange trees. Crop Protection 29(3):300-305.

Benton, J. 1997. Plant Nutrition Manual. Florida, USA, CRC Press LLC. 230 p.

Buol, S. 2003. Soil genesis and classification. 5th ed. Ames, Iowa, Iowa State Press. 494 p.

Cabalceta, G; Molina E. 2006. Niveles críticos de nutrimentos en suelos de Costa Rica utilizando la solución extractora Mehlich 3. Agronomía Costarricense 30(2):31-44.

Czeschlik, D. 2005. Nutrient acquisition by plants. Berlin, Alemania, Springer. 359 p.

Da Costa, L; Guedes, İ; Morris, L; De Oliveira, D. 2005. Biogeochemisty of silica phytoliths in agricultura. Uberlándia, Brasil. III Silicon In Agriculture Conference. $151 \mathrm{p}$.

Datnoff, L; Elmer, W; Huber, D. 2007. Mineral nutrition and plant disease. St Pail. USA, American Phytopathological Society - Press. 278 p.

Epstein, E. 1999. Silicon. Annual Review of Plant Physiology and Plant Molecular Biology 50(1):641-664.

Fauteux, F; Rémus, B; Menzies, J; Bélanger, R. 2005. Silicon and plant disease against pathogenic fungi. FEMS Microbiology Letters 249(1):1-6.

Fregoni, M. 1986. Some aspects of epigen nutrition of grapevines. First International Symposium of Foliar Fertilization by Shering Agrochemical Division. Berlin, Alemania. p. 205-210.

Garbanzo, G; Molina, E; Cabalceta, G; Serrano, E; Ramírez F. 2018. Evaluación de Si aplicado al suelo en el crecimiento, absorción y severidad de enfermedades en vivero de palma aceitera. Agronomía Costarricense 42(1):91-114.

Hardy, F. 1970. Suelos tropicales: pedología tropical con énfasis en América. México, Herrero Hermanos, 270 p.

Jaramillo, D. 2011. El Suelo. Origen, propiedades, espacialidad. Medellín, Colombia, Universidad Nacional de Colombia. p. 17-38.

Klein, C; Hurlbut C. 2003. Manual de mineralogía. 4ed. Barcelona, España, Reverte S. A. 679 p.

Lambers, H; Stuart, F; Pons, T. 1998. Plan Physiological Ecology. New York, USA, Springer. 539 p. 
Liang, Y; Nikolic, M; Bélanger, R; Gong, H; Song, A. 2015. Silicon in agriculture. From theory to practice. New York, USA, Springer. $250 \mathrm{p}$.

Lizcano, A; Herrera, M; Santamarina, J. 2006. Suelos derivados de cenizas volcánicas en Colombia. Rev. Internacional de Desastres Naturales, Accidentes e Infraestructura 6(2):167-198.

Lobo, A. 2013. Validación de un protocolo para la determinación de silicio ( $\mathrm{Si}$ ) en muestras de suelo y tejidos vegetales. Tesis Lic. San José, Costa Rica, Universidad de Costa Rica. 95 p.

Ma, J. 2015. Silicon. Handbook of plant Nutrition. 2 ed. Chapter 20. Florida, USA, CRC press. p. 681-696.

Ma, J; Yamaji, N. 2006. Functions and transport of silicon in plants. Cellular and molecular life sciences 65(1):3049-3057.

Malavolta, E. 1986. Foliar fertilization in Brasil. Present and perspectivas. International Symposium of Foliar Fertilization by Schering Agrochemical Division. Berlin, Alemania. p. 173-192.

Marafon, A; Endres, L. 2013. Silicon: Fertilization and nutrition in higher plants. Amazonian Journal of agricultural and environmental sciences 56(4):380-388.

Marschner, P; Marschner, H. 2012. Marschner's mineral nutrition of higher plants. 3rd ed. London, England, Elsevier/Academic Press. 651 p.

Mengel, K; Kirkby, E; Kosegarten, H; Apple, T. 2001. Principles of plant nutrition. 5 ed., Netherlands, Kluwer Academic Publishers. 851 p.

Nath, G. 2015. Regulation of nutrient uptake by plants. A biochemical and molecular approach. New York, USA, Springer. 209 p.

Ortiz, R; Fernández, O. 2000. Cultivo de palma aceitera. San José, Costa Rica, EUNED. 208 p.

Pilon, C; Soratto, R; Broetto, F; Fernández, A. 2014. Foliar or soil applications of silicon alleviate water-deficit stress of potato plants. Agronomy Journal - Crop Ecology y Physiology 106(6):2325-2334.

Pilon, C; Soratto, R; Moreno, L. 2013. Effects of soil foliar application of soluble silicon on mineral nutrition, gas exchange, and growth of potato plants. Crop Science 53(1):1605-1614.

Quero, E. 2008. La Biosilicificación proceso biológico fundamental en la productividad vegetal. Revista de Riego: Protección y Nutrición de Hortalizas y Frutas 6(39):72-76.

Ramírez, F; Muñoz, F. 2010. Curva de nutrientes para la etapa de vivero de tres materiales de palma aceitera (Elaeis guineensis Jacq.) (en línea). Congreso ecuatoriano de la ciencia del suelo. Santo Domingo, Ecuador. Consultado 12 feb. 2018. Disponible en http://www.
secsuelo.org/wp-content/uploads/2015/06/2.-FloriaRamirez.-Curva-materiales-vivero.-PALMATICACosta-Rica.pdf

Rodríguez, R; Jiménez, E. 2013. Procedimiento para la evaluación de medidas de crecimiento de la hoja 1 y muestreo foliar en plantas de vivero. Boletín Mensual de la Unidad de Investigación de Palma Tica S.A. y Cukra Development Corporation. San José, Costa Rica. 8 p.

Salim, B; Ei-Gawad, A; EI-Yazied, A. 2014. Effect of foliar spray of different potassium sources on growth, yield and mineral composition of potato (Solanum tuberosum L.). Middle East Journal of Applied Sciences 4(4):1197-1204.

Savant, N; Snyder, G; Datnoff, L. 1997. Silicon management and sustainable rice production. Adv. in Agr. 58:151-199.

Seron, H. 2004. Características agronômicas e eficiência de fontes de silício. Universidad Federal de Goiás. Jataí, Brasil. 13 p.

Sidhu, M; Surianto, Z; Sinuraya, Z. 2001. Investigations into corrective treatment of micro-nutrient deficiency in a peat soil oil palm nursery. Planter, Kuala Lumpar 77(1):7-8.

Sposito, G. 2008. The chemistry of soil. 2 ed. USA, Oxford University Press. 344 p.

Taiz, L; Zeiger, E. 2006. Fisiología Vegetal. 3 ed. Castellón, España, Universitat Jaume. 583 p.

Trinidad, A; Aguilar D. 1999. Fertilización foliar un respaldo importante en rendimiento de los cultivos. Revista Terra 17(3):247-255.

Tsai, S; Filho, F; Lima, G; Fraggiani, E; Salomao, R; Martinati, J; Guzzo, S; Staffocker, A. 2004. Interações do silício com patógenos e resistência sistémica induzida em plantas. III Silicon In agriculture Conference, Uberlándia, Brasil. 151 p.

Ulloa, M; Ramírez, F. 2011. Resultado del diagnóstico sobre la necrosis foliar del vivero Coto 49. Boletín Mensual de la Unidad de Investigación de Palma Tica S.A. y Cukra Development Corporation, Costa Rica. 3 p.

Ulloa, M; Serrano, E. 2012. Prueba de sensibilidad in vitro de Colletotrichum gloeosporioides y Curvularia lunata, principales agentes causales del Complejo de Necrosis Foliar (CNF) a diferentes fungicidas. Boletín Mensual de la Unidad de Investigación de Palma Tica S.A. y Cukra Development Corporation, Costa Rica. 9 p.

Yoshida, S. 1965. Chemical aspects of the role of silicon in physiology of rice plant. Bulletin of the National Institute of agricultural Science 15(1):1-58.

Zapata, H. 2004. La química de la acidez del suelo. Medellín, Colombia, Sociedad Colombiana de la Ciencia del Suelo. 207 p. 